\title{
Familial Hypokalemic Periodic Paralysis with Myotonic Phenomenon : Case Report
}

\author{
Longo NM*, Pellegrinelli A, Barbosa DG, Almeida IT, Pessoa LFO, Schoeps VA, Oliveira FTM, Rocha \\ CMG, Tilbery CP and Sanvito WL
}

Irmandade Santa Casa de Misericórdia de São Paulo, Vila Buarque, São Paulo, Brazil

*Corresponding author: Longo NM, Irmandade Santa Casa de Misericórdia de São Paulo, Rua Dr Cesario Motta Jr., n.112, Vila Buarque, São Paulo, CEP: 01221-020, Tel: 5511955588525, E-mail: nataliamatalongo@ gmail.com

Citation: Longo NM, Pellegrinelli A, Barbosa DG, Almeida IT, Pessoa LFO, et al. (2017) Familial Hypokalemic Periodic Paralysis with Myotonic Phenomenon : Case Report. J Neurol Neurol Disord 3(2): 205. doi: 10.15744/2454-4981.3.205

Received Date: December 20, 2016 Accepted Date: August 22, 2017 Published Date: August 25, 2017

\begin{abstract}
Hypokalemic Periodic Paralysis (HypoPP) is a rare disease whereby voltage-gated ion channels are mutated and it is characterized by episodic flaccid paralysis concomitant to variations in blood potassium levels. Attacks usually happen after exercise or high carbohydrate meals. The diagnosis is made with laboratory data wich helps to exclude other causes and confirm low potassium serum or myotonia on eletromyography (EMG). The case report shows a patient who had severe symptoms of HypoPP and had laboratory data of low potassium serum during attacks and EMG with features more common in Hyperkalemic Periodic Paralysis. It also presented a great reponse to therapeutic treatment with potassium reposition and acetazolamide.
\end{abstract}

Keywords: Hypokalemic Periodic Paralysis; Episodic Flaccid Paralysis; Blood Potassium Levels, Myotonia

\section{Introduction}

Neurological channelopathies represent a rapidly expanding group of diseases that illustrate the challenges and opportunities in investigating the pathogenesis of rare diseases [1]. Familial periodic paralysis was recognized as clinical entity as early as the end of the nineteenth century. A decreased in blood potassium levels during attacks of periodic paralysis was described in 1934 by Biemond and Daniels, and founded the basis for the first classification of periodic paralysis $[2,3]$.

Hypokalemic Periodic Pafralysis (hypoPP) is an autossomal dominant neuromuscular disease whereby voltage-gated ion channels are mutated, resulting in abnormalities of sarcolemmal excitation. The more common mutated channel are calcium or sodium, less frequently potassium. Seventy to $80 \%$ of the cases are caused by mutation of CACNA1S and 10\% by mutations of SCN4A [4,5].

Although the genetic defect is present throughout life, the mean age at onset is in the second decade. The frequency of attacks is higher from the second to the fourth decades of life, and then tends to decrease [6].

The prevalence of hypoPP has been estimated at 1:100,000 [6]. This rare disease is characterized by episodes of flaccid skeletal muscle paralysis accompanied by reduced serum potassium level [5]. The disease typically manifests as bouts of mild to severe muscle weakness lasting hours and sometimes days associated with hypokalemia triggered most commonly by rest after exercise, high carbohydrate meals and cold temperatures [4,7]. Some cases may present as sporadic because of the incomplete penetrance of the disease, mostly in women [6]. The interval between crises may be prolonged by prevent treatment with potassium salts or acetazolamide [8].

A variable myopathy develops in at least $25 \%$ of affected individuals and may result in a progressive fixed muscle weakness that manifests at variable ages as exercises intolerance predominantly in the lower limbs. It may occur independent of paralytic symptoms and may be the sole manifestation of hypoPP [8]. The disease is potentially lethal and a mortality of $10 \%$ was common before the introduction of intensive care unit [6]. 


\section{Case Report}

A 39 years-old-woman, afro descendant, with hypertension and depression history, was admitted in this hospital with a history of muscle pain and weakness.

When the patient was 9 years-old, she started to present intermitently pain in upper and lower limbs, which were worse at night, making difficult to sleep. After 7 years, she started to have syncope episodes. In 2007, she presented paralysis episodes after exercise.

In 2011, the patient went on a trip where she started to present weakness and diffuse myalgia. This symptoms evoluate with gait disturbance and difficult to speak. She went several days to the emergency.

One day, she was admitted in the hospital with respiratory insuffiency and she was intubated. During hospitalization, she presented four cardiac arrest and a low potassium serum level of 1.7. She was investigated with an electromyography (EMG)and a muscle biopsy. The EMG had the presence of myotonia and high frequency muscle action potential. The signs of myotonia were found just at the EMG, but not during examination. The muscle biopsy showed variation in the muscle fiber caliber,nucleo center and degeneration fibers. The immunohistochemical study had CD4 and MHCn positivity (Figure 1). These suggested inflamatory activity abnormality.

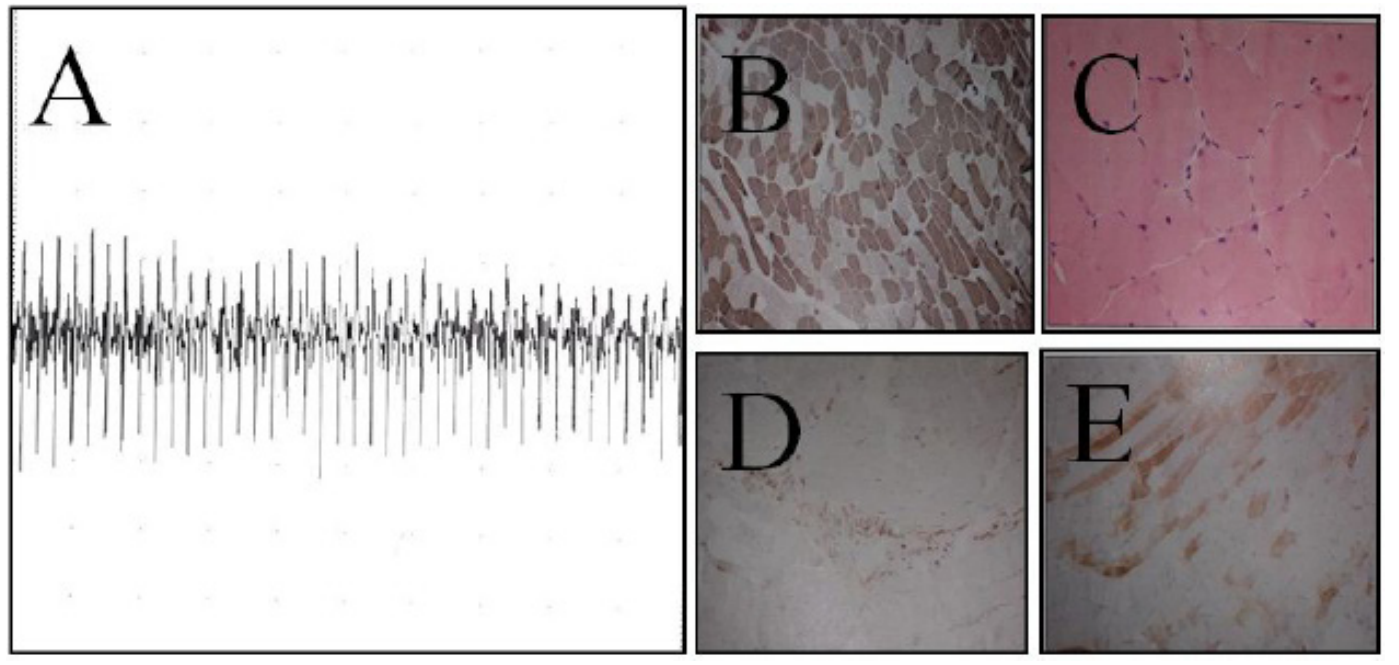

Figure 1: (A) EMG which shows myotonia (B) Muscle biopsy with ATPase stain (C) Muscle biopsy with hematoxylin and eosin stain (D) Muscle biopsy with immunohitochemical for CD4 antibodies (E) Muscle biopsy with immunohistochemical for MHCn antibody.

The patient was treated with intravenous potassium reposition with $3 \mathrm{mEq} / \mathrm{kg} /$ day for 5 days until the potassium level was normalized and acetazolamide $250 \mathrm{mg} 3$ times a day. After 5 days, the patient started oral reposition of potassium with $12 \mathrm{mEq} 3$ times a day and after 25 days, shewas discharded from the hospital with acetazolamide $250 \mathrm{mg} 3$ times a day and potassium $6 \mathrm{mEq}$ 3 times a day. She had a great response to the treatment, even though she was still feeling weak, she was talking and walking at the time of the discharged from the hospital.

\section{Discussion}

Familial hypokalemic periodic paralysis is inherited in an autosomal dominant manner and it is common in Caucasians. Most individuals with this disease have an affected parent [2,9]. HypoPP has a reduced penetrance and expression in women. Women may even escape expression of the phenotype, although they transmit the disease. In consequence, a male to female predomiance of 3:1 has been reported. The penetrance of hypoPP was found to be $80 \%$ in women and $100 \%$ in men [8]. Affected women tend to have fewer attacks than affected men, reflecting the decreased expressivity of the disease in women [6]. As in our case report, the patient was a female, afro descendant with a not known affected parent.

The age of onset of hypoPP is within the second decade of life: $60 \%$ of patients develop the disease before the age of 16 , although younger or later onsets have been reported [3]. The patient of this case report started symptoms when she was 9 year-old.

HypoPP is a heterogenous disease feature by episodic muscle paralysis associated with ictal hypokalemia during the period of attacks. Serum potassium levels are usually normal in HypoPP patients at the baseline [10]. Typically, attacks occur at night and the patient awakes paralyzed [3]. Attacks commonly occur several hours after strenuous exercise or a carbohydrates rich meals. The ocular, oropharyngeal and respiratory muscles are usually spared, but may be affected in the most severe attacks [6].

There are two types of hypoPP: the paralytic and myopathic form. The paralytic form muscles lose the ability to contract and the sensation remains intact. The myopathic form the patient presents slow progressive fixed muscle weakness that begins as exercise intolerance [11]. In a case report described by Tae-Hwan Kil and June-Bum Kim, a 16 year old boy had just sundden episodes of 
breathing difficulty and cyanosis. Different from our patient who had muscle weakness fluctuancy and an episode of respiratory insufficiency and cardiac arrest.

The episodic symptoms of hypoPP can be life-threating. It can affectrespiratory muscle as it was reported in some experiments and it can provoke cardiac arrhythmias [12,6]. Respiratory and cardiac symptoms in hypoPP happened by severe hypokalemia as it is demonstrated in our case report.

We recognize an attack by the generalized weakness of the limbs without other neurologic findings, the common precipitants to an attack, and the laboratory data that help exclude other causes and confirm characteristic features such as low potassium or myotonia on EMG [13]. The EMG shows a typical myotonic discharge which has a waxing and waning quality called "divebomber". Myotonia on EMG occurs in approximately 75\% of individuals with HyperPP, but it not occur in HypoPP [11]. The work of Sugira et al. reporteda mutation, P1158S, of the sodium channel gene in a family with cold-induced HypoPP and myotonia. Their resultsindicated that the hypokalemic paralysis in cold atmospheric temperatures is due to hyperpolarizing shifts of the gating property of the mutante sodium channel. Another feature is that the channel has a slower time of inactivation, which has been reported in most myotonia-related mutant sodium channel [7]. This case shows that HypoPP can present myotonia on EMG, even though it is more common in HyperPP cases.

Muscle biopsy showed vacuolar myopathy in $67 \%$ of patients with mutations. In patients without mutation, muscle biopsy showed either myopatic change (50\%) or was normal (50\%) [13]. The muscle biopsy in our case showed inflamatory activity abnormality.

The myopathy arises independently of the frequency and the severity of the attacks. It can even occur in the absence of attacks. The onset is usually in the fourth or fifth decade of life. The myopathy affects mostly the muscle of the pelvic girdle and the proximal muscle of upper and lower limbs [6].

Typically, weakness recovers when serum potassium normalizes [4]. Permanent muscle weakness is rarely but possibly observed in young adult age. The fixed muscle weakness is more common in older patients as well as the lack of reponsiveness to treatment $[6,4]$. Permanent weakness may indicate a state of persistent or continuously recurring mild attacks and may be dramatically improved by long term use of carbonic anhydrase inhibitors [6].

Acetazolamide has been the most common treatment choice for hypoPP for nearly 50 years. HypoPP is caused by a mutations in 1 of 2 sarcolemmal ion channel genes, CACNA1S and SCN4A. Since the discovery of the 2 causative genes, it has been occasionally reported that some patients with SCN4A mutations associated with hypoPP reacted adversely to acetazolamide, but other reports have indicated benefit [5]. This case report showed a great reponse to the treatment with acetazolamide and potassium reposition.

\section{Conclusion}

We conclude that the patient of this case report started with symptoms before the aged 16 years old. She presented weakiness after exercise and carbohydrates rich meals. She felt worse at night, having difficulty to sleep. All these symptoms are typical of hypoKK.

The respiratory insufficiency and cardiac arrest are rare symptoms presented by the patient of hypoPP.

The myotonia on EMG is common on hyperPP and this case report showed it is possible in hypoPP.

Our case report findings suggest that this patient presents symptoms of myopathy during periods of low potassium serum wich has a great response of potassium supplementation and acetazolamide and it was diagnosed with EMG and muscle biopsy suggesting a HypoPP of sodium channel variant, which is a rare disease.

\section{References}

1. Jen JC, Ashizawa T, Griggs RC, Waters MF (2016) Rare neurological channelopathies - networks to study patients, pathogenesis and treatment. Nat Rev Neurol 12: $195-203$

2. Venance SL, Cannon SC, Fialho D, Fontaine B, Hanna MG, et al. (2006) The primary periodic paralyses: diagnosis, pathogenesis and treatment. Brain 129: 8-17.

3. Fontaine B, Lapie P, Plassart E, Tabti N, Nicole S, et al. (1996) Periodic paralysis and voltage-gated ion channels. Kidney Int 49: 9-18.

4. Levitt JO (2008) Practical aspects in the management of hypokalemic periodic paralysis. J Transl Med 6: 18.

5. Matthews E, Portaro S, Ke Q, Sud R, Haworth A, et al. (2011) Acetazolamide efficacy in hypokalemic periodic paralysis and the predictive role of genotype. Neurology 77: 1960-4.

6. Fontaine B (2008) Periodic Paralysis. Adv Genet 63: 3-18.

7. Sugiura Y, Makita N, Li L, Noble PJ, Kimura J, et al. (2003) Cold induces shifts of voltage dependence in mutant SCN4A, causing hypokalemic periodic paralysis. Neurology 61: 914-8.

8. Vicart S, Stemberg D, Arzel-Hézode M, Franques J, Bendahhou S, et al. (2002) Hypokalemic Periodic Paralysis. Gene Rev 1-40.

9. Garg RK, Malhotra HS, Verma R, Sharma P, Singh MK (2013) Etiological spectrum of hypokalemic paralysis: A retrospective analysis of 29 patients. Ann Indian Acad Neurol 16: 365-70

10. Cheng CJ, Kuo E, Huang CL (2013) Extracellular Potassium Homeostasis: Insights from Hypokalemic Periodic Paralysis. Semin Nephrol 33: 237-47.

11. Turcotte J, White D, Tilney PVR (2013) Hypokalemic Periodic Paralysis: Two Cases of Profound Weakness. Air Med J 32: 181-3. 
12. Kil TH, Kim JB (2010) Severe respiratory phenotype caused by a de novo Arg528Gly mutation in the CACNA1S gene in a patient with hypokalemic periodic paralysis. Eur Paediatr Neurol 14: 278-81.

13. Miller TM, Dias da Silva MD, Miller HA, Kwiecinski H, Mendell JR, et al. (2004) Correlating phenotype and genotype in the periodic paralyses. Neurology 63: $1647-55$

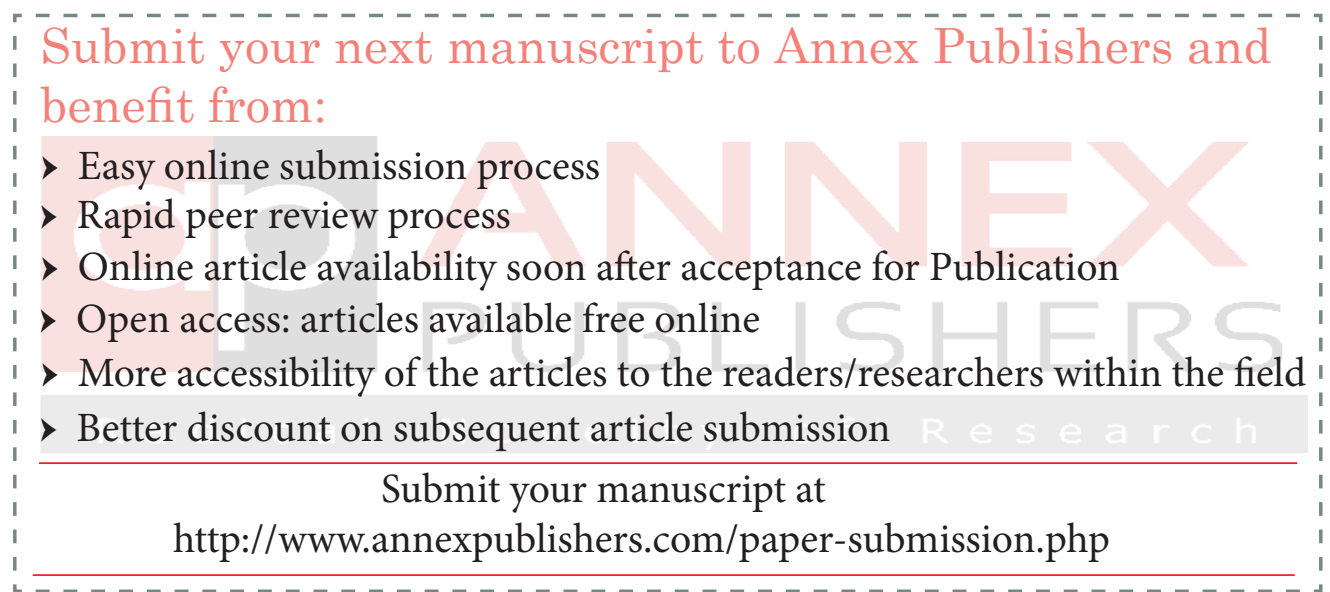

